$\xi=$

\title{
Screening of efficient phosphate solubilizing fungi from mine soil and effect of phosphofungi on seed germination and vigour index of ground nut (arachis hypogaea $\mathrm{L}$.) and green gram (vigna radiata $\mathbf{L}$.)
}

\author{
Lokesh Shanubhoganahalli Thimmappa, Naveen Kumar Kudure Jayanna*, Thippeswamy Basaiah \\ Department of P.G. Studies and Research in Microbiology, Bioscience Complex, Kuvempu University, \\ Jnana Sahyadri, Shankaraghatta-577 451, Shivamogga District, Karnataka, India \\ *Corresponding author E-mail: naviekj@gmail.com
}

\begin{abstract}
Background: The use of chemical fertilizers to solve the problem of nutrient deficiency in soil has been associated with a number of environmental problems.

Objective: The aim of this study was to perform an isolation and screening of native phosphofungi from mine soil. To evaluate the effect of phosphofungi on seed germination and seedling vigour index of Ground nut and Green gram.

Materials and Methods: The phosphofungi were screened using Pikovskaya's agar medium with tricalcium phosphate $\left(\mathrm{Ca}_{3}\left(\mathrm{PO}_{4}\right)_{2}\right)$ as the phosphorus source. The soluble phosphorus, Phosphate solubilizing activity, titrable acidity, $\mathrm{pH}$ and fungal biomass were determined. Mineral phosphate solubilizing (MPS) activities of fungal isolates were tested in tricalcium phosphate medium.

Results: Five promising phosphate solubilizing fungal species were screened. Aspergillus niger $(13 \pm 1.0 \mathrm{~mm})$ and Aspergillus flavus $(8 \pm 0.6 \mathrm{~mm})$ showed the more phosphate solubilising index than compared to other fungal isolates. Analyzing the possible phosphorus released, from $3^{\text {rd }}, 6^{\text {th }}, 9^{\text {th }}$, and $12^{\text {th }}$ day of incubation. Antagonistic effect of phosphofungi Aspergillus flavus (65 $\left.\pm 11 \mathrm{~mm}\right)$ and Aspergillus niger $(60 \pm 13 \mathrm{~mm})$ showed more effective against Colletotrichum sp. than compared to other fungal isolates. Aspergillus flavus $(745 \pm 20 \mu \mathrm{g} / \mathrm{ml})$ and Penicillium spinulosum $(600 \pm 20 \mu \mathrm{g} / \mathrm{ml})$ showed maximum phosphate solubilizing activity on $3^{\text {rd }}$ and $12^{\text {th }}$ day of incubation. Fusarium redolens showed the more effectiveness on seed germination and seedling vigour than compared to other fungal isolates. Aspergillus flavus showed positive result for the production of Indole 3 acetic acid and remain four fungal strains are negative result.

Conclusion: Biofertilizers from native phosphofungi could be used alongside reduced levels of inorganic fertilizers to enhance soil available Phosphorous.
\end{abstract}

Keywords: Arachis Hypogaea; Mine Soil; Phosphofungi; Phosphate Solubilizing Activity; Vigna Radiata.

\section{Introduction}

Phosphorous is second vital nutrient next to nitrogen required for growth of plants and microorganisms. But most of the phosphorous is not available to plants. Only $1-2 \%$ phosphorous is supplied to above ground parts of the plants (Ashok et al., 2012; Anjoram and Mohammed, 2014; Elias et al., 2016). Therefore, to meet out the phosphorous demand of the plants, exogenous source of phosphorous is applied to plants as chemical fertilizers. One of the most common forms of phosphate fertilizer is super phosphate (single/triple). The basic raw material for phosphate fertilizer is the rock phosphate. But rock phosphate is not recommended to apply directly due to agronomic problems being as raw material (Karunai et al., 2011; Baig et al., 2012; Gupta et al., 2012).

So, the phosphate solubilizing microbes convert these insoluble phosphates into soluble forms through special mechanism. That is they carry out the process of acidification, chelation, exchange reactions and production of gluconic acid. Many of the isolates are evidence for the presence of multiple organic acids. They are able to produce thirteen kinds of organic acids including citric acid gluconic acid, 2-keto-gluconic acid, succinic acid, glycolic acid, lactic acid, fumaric acid, formic acid, acetic acid, butyric acid, isobutyric acid, valericacid, and isovaleric acid (Srilatha and Venkateshwarlu, 2009; Naik et al., 2013; Kumar et al., 2014). The type of organic acid produced and their amount differ with different organisms. Among them glucoronic acid and 2-keto-gluconic acid seems to be the most frequent agent of mineral phosphate solubilization. Other organic acids such as acetic, citric, succinic, propionic, glycolic, oxalic, malonic, fumaric and tartaric acid, amino acid, vitamins and growth promoting substances like indole-3-acetic acid (IAA) and gibberellic acid which helps in better growth of plants etc. Have also been identified among phosphate solubilises (Morales et al., 2011; Nisha et al., 2014). Carboxylic acids are more effective as compared to monobasic, aromatic acids and aliphatic acids and then to phenolic, citric, and fumaric acids (Kulkarni et al., 2011; Anjalisoni et al., 2013).

Some phosphate solubilizing fungi such as Aspergillus, Penicillium, Fusarium and Sclerotium from fungal genera is the most powerful phosphate solubilisers (Pradhan and Sukla, 2005; Sem- 
biring et al., 2015). Several species of fungi are able to convert insoluble metal compounds example certain oxides and phosphates into soluble forms by the excretion of protons and/or various metabolites, including organic acids (Priya et al., 2013). Fungal solubilization of insoluble metal compound appears to be of increasing biotechnological potential, for example in the winning of metals from low grade ores and recycling of metals from industrial by products (Manikandan and Muthuselvam, 2014).

The fungi possess greater ability to solubilize insoluble phosphate than bacteria. Species of Aspergillus, Penicillium and Fusarium have been widely mentioned as efficient strains of phosphate solubilizers (Doni et al., 2014; Pooja et al., 2015).

Present study was taken up to isolate and characterize the phosphate solubilizing fungi from mine soil and also studied the effect of phospho fungi on seed germination and seedling vigour index of Ground nut and Green gram.

\section{Material and methods}

\subsection{Collection of mine soil}

Rhizosphere soil sample was collected at $15-20 \mathrm{~cm}$ depth of mine soil Bellary, Karnataka, India during the 2014-2015.The samples were taken by means of sterilized spatulas and collected in sterile sealed polythene bags. The sample was brought to the laboratory and was maintained at room temperature for microbiological study.

\subsection{Isolation of fungi by serial dilution method}

One gram of soil sample was taken in a conical flask containing nine milliliters of sterile distilled water and shaken well in vortex mixer for 30 minutes. From this stock, various dilutions were prepared from $10^{-1}$ to $10^{-7}$, using sterile distilled water. One milliliters of the diluted sample was poured into Petri plates containing the Pikovskaya's agar medium and Potato dextrose agar (PDA) medium. Distinct fungal colonies grown in Pikovskaya's agar medium and Potato dextrose agar medium were isolated from repeated plating (Aneja, 2001; Paul and Daniel, 2007; Naveenkumar et al., 2012).

\subsection{Pure cultures of phosphate solubilizing fungal spe- cies}

Positive fungal colonies were subculture, on fresh Petri plates containing medium for plate assay. Fungal cultures were isolated by incubating the plates in inverted position in incubator for 3 to 5 days at $28{ }^{\circ} \mathrm{C}$. Positive cultures were screened by observing transparent halo zones in Pikovskaya's medium, which is due to the solubilization of insoluble tricalcium phosphate into the soluble form and halo zones appears on the medium which is due to the production of organic acids leading to lowering of $\mathrm{pH}$ within the medium (Aneja, 2001)

\subsection{Identification of fungi}

Fungal morphology was studied macroscopically by observing colony features (colour and surfaces) under Stereo binocular microscope and microscopically by staining with Lacto phenol cotton blue and observed under binocular compound microscope for the conidia, conidiophores and arrangement of spores. All the isolates were pure cultured, identified and studied for Phosphate solubilizing activity (Funder, 1961; Subramanian, 1993).

\subsection{Phosphate solubilization by qualitative method}

All the suspected colonies from PVK medium were subjected to spot inoculative individually at the centre of PVK medium. The plates were incubated at $28-30{ }^{\circ} \mathrm{C}$ up to 7 days. The diameter of clear zone was measured as follows; the Phosphate solubilization index is the ratio of total diameter, Zone of clearance $(\mathrm{Z})$ and the colony diameter (C) (Malviya et al., 2011).

$$
\text { SI }=\text { Colony diameter + clearing zone }
$$

\subsection{Quantitative measurement of phosphate solubiliza- tion}

The efficacies of the selected five fungal cultures in the solubilization of insoluble phosphates (TCP) were determined. These cultures were grown in $100 \mathrm{ml}$ of Pikovskaya's liquid medium containing $250 \mathrm{mg}$ of TCP as the phosphate source for 12 days at $28 \pm$ $30{ }^{\circ} \mathrm{C}$ in a shaker. Every three days the sample was taken and filtered using Whatman No.1 filter paper. The biomass of the fungus was measured on dry weight basis and the $\mathrm{pH}$ of the filtrate was measured with a digital $\mathrm{pH}$ meter. From filtrate, the phosphate was measured by the method of Fiskay-Subbarao (Sadasivam and Manickam, 1996; Aneja, 2001)

\subsection{Screening for indole-3-acetic acid production (IAA)}

The potent phosphofungi will be grown in potato dextrose medium and will grown culture will be centrifuged at $3000 \mathrm{rpm}$ for 30 minutes supernatant $(2 \mathrm{ml})$ will be mixed with 2 drops of orthophosphoric acid and $4 \mathrm{ml}$ of salkwoski reagent $(50 \mathrm{ml}$ of $35 \%$ perchloric acid $1 \mathrm{ml} 0.5 \mathrm{ml} \mathrm{FeCl} 3$ solution). Development of pink colour after 30 minutes to 2 hour incubation at room temperature indicates indole-3-acetic acid production (Kafrawi et al., 2014).

\subsection{Measurement of $\mathrm{pH}$ change and titrable acidity}

One $\mathrm{ml}$ of 3 days old culture $\left(1 \times 10^{3} \mathrm{CFU}\right)$ in sterile distilled water will be added to sterile Potato dextrose broth medium in $250 \mathrm{ml}$ conical flask and incubated on rotary shaker for 7 days at room temperature. Sterile uninoculated medium will be served as control. Initial $\mathrm{pH}$ and change in $\mathrm{pH}$ will be noted each day for one week by digital $\mathrm{pH}$ meter. The titrable acidity of culture media of each day culture filtrate will be estimated. For this, culture filtrate will be centrifuged at $1000 \mathrm{rpm}$ for 10 minutes. Five ml of supernatant will be added with few drops of phenolphthalein indicator and titrated against $\mathrm{NaoH}$ Consumed $(0.01 \mathrm{~N}) / 5 \mathrm{ml}$ of culture filtrate (Ponmurugan and Gopi, 2006)

\subsection{Screening of antagonistic efficiency of phosphorus fungi by dual culture plate method}

Colony interaction between phosphofungi and test pathogens will be studied in vitro by dual culture plate method. The individual test organism (plant pathogens) will be grown separately on potato dextrose agar medium and the individual species of phosphorus fungi will be grown in separately on potato dextrose agar medium respectively. The point inoculation of the individual species of fungi and test pathogen will be inoculated just opposed to each other approximately $3 \mathrm{~cm}$ apart, on potato dextrose agar medium in Petri plates. The position of colony margin will be recorded daily. Assessment will be made for the fungi against test organism. When it achieved an equilibrium after which there will be no further alteration in the growth, the assessment will be done by the formula (Baig et al., 2012; Kannahi and Umaragini, 2013).

Growth of organism in control plate X 100 Percentage of inhibition $=$ Growth of organism in dual culture plate 


\subsection{Effect of phosphofungi on seed germination and seedling vigour}

Germination test was conducted using blotting paper (BP) method of germination and fifty seeds per replication were sown on paper towel. Germination test was conducted according to the International Seed Testing Association rules. Seeds were placed on the surface of double sheets of paper towel which were moistened with distilled water. The seeds were covered with other sheet of paper towel. The sheets were rolled and placed vertically in a plastic beaker, covered with polythene bags and placed at $30{ }^{\circ} \mathrm{C}$ temperature in a germinator. Germination was interpreted as the percentage of seeds producing normal seedlings (Kapri and Tewari, 2010).

Number of seed germination $\mathrm{x} 100$

$\%$ of seed germination $=-$ -

Seedling vigour $=($ Average root length + Average shoot length $) \mathrm{x}$ $\%$ of seed germination

\subsection{Statistical analysis}

All the results were statistically analyzed using SPSS software to determine the mean of three replicates and its standard error value from independent experiments.

\section{Results}

\subsection{Isolation and screening of phosphate solubilizing fungi by serial dilution method}

In the present study, five different phosphate solubilizing fungal isolates were screened from mine soil by Serial dilution method such as Aspergillus niger, Aspergillus flavus, Fusarium redolens, Penicillium spinulosum and Penicillium sp. (Figure 1) (Table 1).

Table 1: Isolation and Characterization of Phosphate Solubilizing Fungi from Mine Soil

\begin{tabular}{ll}
\hline Sl. No. & Name of the Fungal Species \\
\hline 1 & Aspergillus niger \\
2 & Aspergillus flavus \\
3 & Fusarium redolens \\
4 & Penicillium spinulosum \\
5 & Penicillium sp. \\
\hline
\end{tabular}

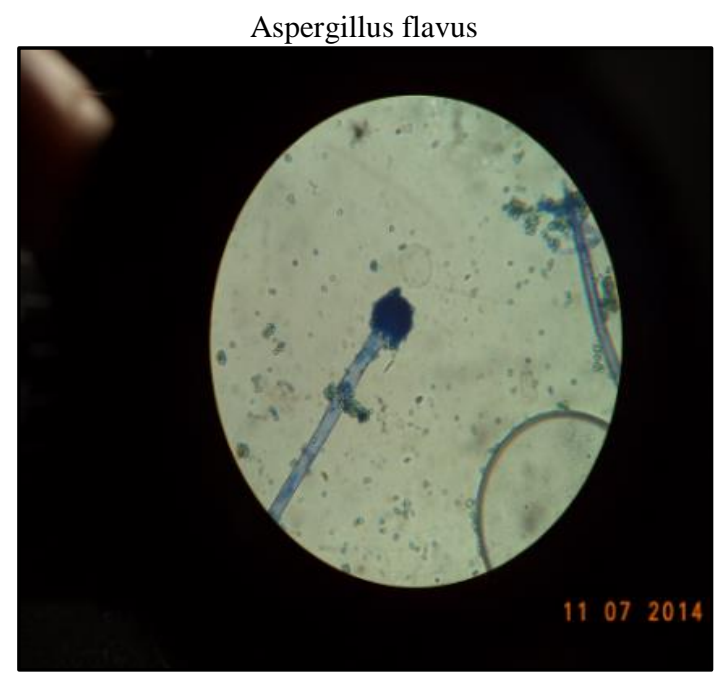

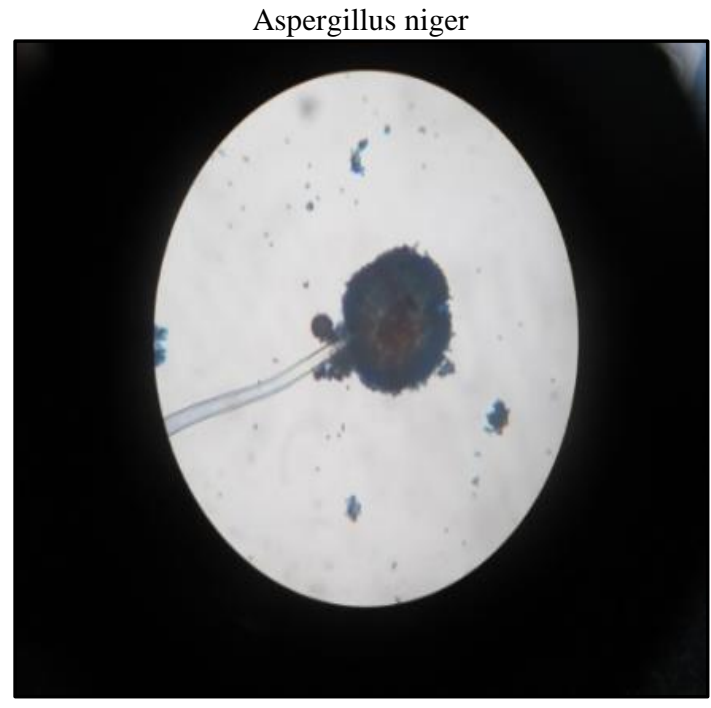

Penicillium sp.

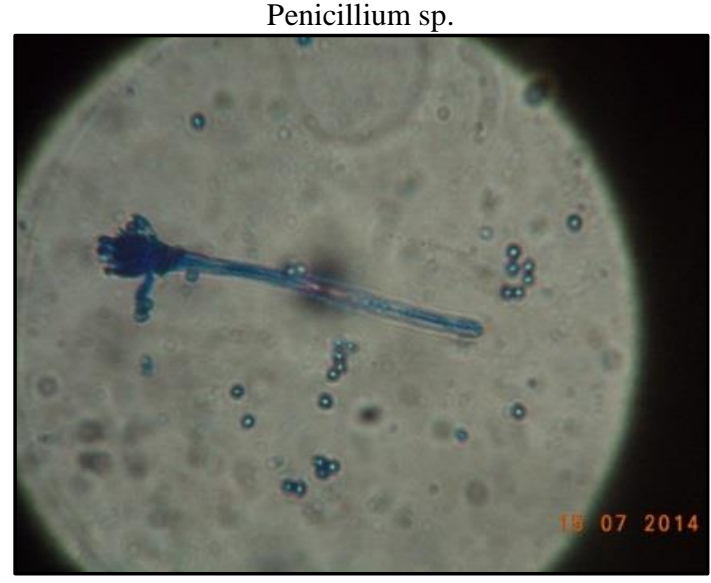

Fusarium redolens

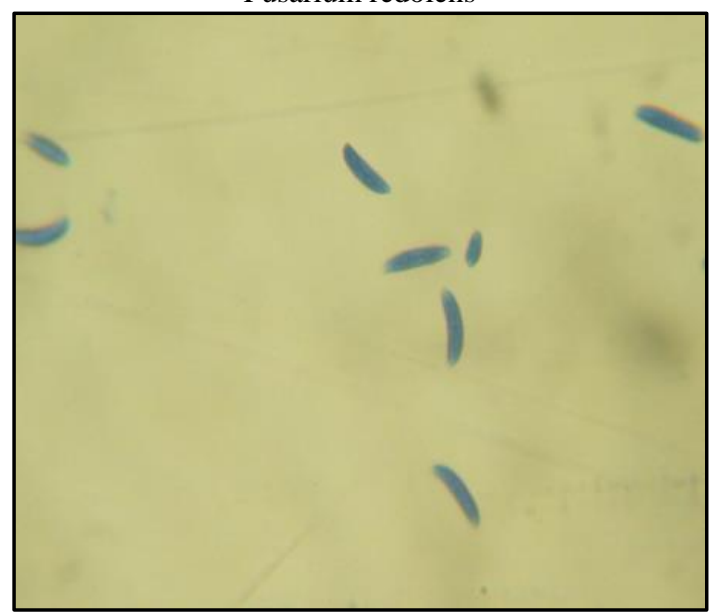

Fig. 1: Microscopic View of Different Phosphate Solubilizing Fungal Species Isolated from Mine Soil

\subsection{Estimation of phosphate solubilizing index (SI)}

The results of the phosphate solubilizing activity of the five fungal species were isolated from the mine soil is given in table-1. As indicated in the table, five fungal isolates, i.e., Aspergillus niger, Aspergillus flavus, Fusarium redolens, Penicillium spinulosum, Penicillium sp. and showed a zone of clearance as well as mycellial growth. Aspergillus niger $(13 \pm 1.0 \mathrm{~mm})$ showed maximum zone of clearance than compared to Aspergillus flavus $(8 \pm 0.6 \mathrm{~mm})$, Fusarium redolens $(1 \pm 0.1 \mathrm{~mm})$, Penicillium spinulosum $(2 \pm$ $0.3 \mathrm{~mm})$ and Penicillium sp $(1 \pm 0.2 \mathrm{~mm})$ (Table 2). 
Table 2: Phosphate Solubilizing Ability of the Selected Five Fungal Species Isolated from Mine Soil in Pikovskaya's Solid Medium

\begin{tabular}{|c|c|c|c|c|}
\hline $\begin{array}{l}\text { Sl. } \\
\text { No. }\end{array}$ & $\begin{array}{l}\text { Name of the or- } \\
\text { ganism }\end{array}$ & $\begin{array}{l}\text { Mycelial } \\
\text { Growth } \\
(\mathrm{mm})\end{array}$ & $\begin{array}{l}\text { Zone of } \\
\text { Clearance } \\
(\mathrm{mm})\end{array}$ & $\begin{array}{l}\text { Total } \\
\text { Diameter } \\
(\mathrm{mm})\end{array}$ \\
\hline 1 & Aspergillus niger & $30 \pm 2.0$ & $13 \pm 1.0$ & $43 \pm 3.0$ \\
\hline 2 & Aspergillus flavus & $10 \pm 1.0$ & $8 \pm 0.6$ & $18 \pm 2.0$ \\
\hline 3 & Fusarium redolens & $7 \pm 0.5$ & $1 \pm 0.1$ & $8 \pm 1.0$ \\
\hline 4 & $\begin{array}{l}\text { Penicillium } \\
\text { spinulosum }\end{array}$ & $3 \pm 0.2$ & $2 \pm 0.3$ & $5 \pm 0.4$ \\
\hline 5 & Penicillium sp. & $2 \pm 0.1$ & $1 \pm 0.2$ & $3 \pm 0.3$ \\
\hline
\end{tabular}

Note: Mean \pm S.E. of three replicates $(n=3)$.

\subsection{Estimation of phosphate solubilizing activity}

In the present study, Aspergillus flavus (745 $\pm 20 \mu \mathrm{g} / \mathrm{ml})$ showed maximum phosphate solubilizing activity on $3^{\text {rd }}$ day of incubation period as followed by Aspergillus niger $(665 \pm 15 \mu \mathrm{g} / \mathrm{ml})$, Fusarium redolens $(560 \pm 10 \mu \mathrm{g} / \mathrm{ml})$ and Penicillium sp. $(460 \pm 15$ $\mu \mathrm{g} / \mathrm{ml})$.

After $12^{\text {th }}$ day of incubation period filtered the Pikovskaya's broth using Whatman No. 1 filter paper for analysing the biomass Aspergillus niger $(1.78 \pm 0.12 \mathrm{gm} / \mathrm{d})$ showed maximum biomass yield on $12^{\text {th }}$ day of incubation period as followed by Aspergillus flavus $(0.60 \pm 0.02 \mathrm{gm} / \mathrm{d})$, Fusarium redolens $(0.52 \pm 0.01 \mathrm{gm} / \mathrm{d})$, Penicillium spinulosum $(0.59 \pm 0.03 \mathrm{gm} / \mathrm{d})$ and Penicillium $\mathrm{sp}$. $(0.55 \pm 0.04 \mathrm{gm} / \mathrm{d})($ Table 3$)$

Table 3: Phosphate Solubilizing Activity of the Selected Five Fungal Species Isolated from Mine Soil in Pikovskaya's Broth

\begin{tabular}{|c|c|c|c|c|c|c|}
\hline \multirow{2}{*}{$\begin{array}{l}\text { SI. } \\
\text { No. }\end{array}$} & \multirow{2}{*}{$\begin{array}{l}\text { Name of the } \\
\text { organism }\end{array}$} & \multicolumn{4}{|c|}{$\begin{array}{l}\text { Phosphate Solubilizing Activity } \\
(\mu \mathrm{g} / \mathrm{ml})\end{array}$} & \multirow{2}{*}{$\begin{array}{l}\text { Biomass } \\
\text { Yield } \\
\text { (gm/days) } \\
12^{\text {th }} \text { day }\end{array}$} \\
\hline & & $\begin{array}{l}3^{\text {rd }} \\
\text { day }\end{array}$ & $\begin{array}{l}6^{\text {th }} \\
\text { day }\end{array}$ & $\begin{array}{l}9^{\text {th }} \\
\text { day }\end{array}$ & $\begin{array}{l}12^{\text {th }} \\
\text { day }\end{array}$ & \\
\hline 1 & $\begin{array}{l}\text { Aspergillus } \\
\text { niger }\end{array}$ & $\begin{array}{l}665 \\
\pm 15\end{array}$ & $\begin{array}{l}355 \\
\pm 20\end{array}$ & $\begin{array}{l}475 \pm \\
8.5\end{array}$ & $\begin{array}{l}185 \pm \\
4.5\end{array}$ & $1.78 \pm 0.12$ \\
\hline 2 & $\begin{array}{l}\text { Aspergillus } \\
\text { flavus }\end{array}$ & $\begin{array}{l}745 \\
\pm 20\end{array}$ & $\begin{array}{l}455 \\
\pm 10\end{array}$ & $\begin{array}{l}570 \pm \\
9.6\end{array}$ & $\begin{array}{l}220 \pm \\
10\end{array}$ & $0.60 \pm 0.02$ \\
\hline 3 & $\begin{array}{l}\text { Fusarium } \\
\text { redolens }\end{array}$ & $\begin{array}{l}560 \\
\pm 10\end{array}$ & $\begin{array}{l}150 \\
\pm 15\end{array}$ & $\begin{array}{l}185 \pm \\
9.8\end{array}$ & $\begin{array}{l}450 \pm \\
15\end{array}$ & $0.52 \pm 0.01$ \\
\hline 4 & $\begin{array}{l}\text { Penicillium } \\
\text { spinulosum }\end{array}$ & $\begin{array}{l}360 \\
\pm 8.0\end{array}$ & $\begin{array}{l}200 \\
\pm 11\end{array}$ & $\begin{array}{l}275 \pm \\
7.8\end{array}$ & $\begin{array}{l}600 \pm \\
20\end{array}$ & $0.59 \pm 0.03$ \\
\hline 5 & $\begin{array}{l}\text { Penicillium } \\
\text { sp. }\end{array}$ & $\begin{array}{l}460 \\
\pm 15 \\
\end{array}$ & $\begin{array}{l}155 \\
\pm 12 \\
\end{array}$ & $\begin{array}{l}295 \pm \\
6.3 \\
\end{array}$ & $\begin{array}{l}600 \pm \\
18 \\
\end{array}$ & $0.55 \pm 0.04$ \\
\hline
\end{tabular}

Note: Mean \pm S.E. of three replicates $(n=3)$.

\subsection{Measurement of $\mathrm{pH}$ change using pikovskaya's broth}

We have studied the $\mathrm{pH}$ variation in different time of incubation using Pikovskaya's broth. In that, five phosphate solubilizing fungal species were used for this study (Table 4).

Table 4:Ph Variation Observed during the Phosphate Solubilizing Activity of the Selected Five Fungal Species Isolated from Mine Soil in Pikovskaya's Broth.

\begin{tabular}{|c|c|c|c|c|c|}
\hline SI. & Name of the & $\mathrm{P}^{\mathrm{H}}$ Val & Numbe & Days) & \\
\hline No. & organism & $3^{\text {rd }}$ day & $6^{\text {th }}$ day & $9^{\text {th }}$ day & $12^{\text {th }}$ day \\
\hline 1 & control & $5.8 \pm$ & $4.33 \pm$ & $5.75 \pm$ & $5.7 \pm$ \\
\hline 1 & control. & 0.3 & 0.3 & 0.5 & 0.4 \\
\hline 2 & Asneroillus niger & $3.8 \pm$ & $4.0 \pm$ & $4.5 \pm$ & $3.15 \pm$ \\
\hline 2 & Aspergginus miger & 0.2 & 0.2 & 0.3 & 0.1 \\
\hline 3 & Asneroillus flavus & $5.4 \pm$ & $5.65 \pm$ & $3.86 \pm$ & $3.17 \pm$ \\
\hline $\mathrm{J}$ & Asperginus Iravus & 0.1 & 0.1 & 0.2 & 0.2 \\
\hline 4 & Fusarium redolens & $5.6 \pm$ & $5.73 \pm$ & $5.89 \pm$ & $6.0 \pm$ \\
\hline 4 & rusantunt Iedoretis & 0.4 & 0.3 & 0.4 & 0.4 \\
\hline 5 & Penicillium & $4.52 \pm$ & $5.79 \pm$ & $6.3 \pm$ & $5.36 \pm$ \\
\hline & spinulosum & 0.3 & 0.4 & 0.3 & 0.4 \\
\hline 6 & Penicillium sp. & $5.69 \pm$ & $5.99 \pm$ & $6.59 \pm$ & $4.36 \pm$ \\
\hline & & 0.2 & 0.1 & 0.2 & 0.3 \\
\hline
\end{tabular}

\subsection{Estimation of titrable acidity}

The present results observed that there was reduction in $\mathrm{pH}$ of the medium but an increase in titrable acidity. This might be due to secretion of organic acids by Phosphate solubilizing fungi. The titrable acidity of the Phosphate solubilizing fungal strains in medium was fluctuated was tabulated in table 5 .

Table 5: Analyze the Titrable Acidity by Phosphate Solubilizing Fungal Species Using Potato Dextrose Broth

\begin{tabular}{lllllll}
$\begin{array}{l}\text { SI. } \\
\text { No. }\end{array}$ & $\begin{array}{l}\text { Name of the } \\
\text { organism }\end{array}$ & $1^{\text {st }}$ day & $2^{\text {nd }}$ day & $3^{\text {rd }}$ day & $\begin{array}{l}4^{\text {th }} \\
\text { day }\end{array}$ & $\begin{array}{l}5^{\text {th }} \\
\text { day }\end{array}$ \\
\hline \multirow{2}{*}{1} & Control & $1.0 \pm$ & $6.3 \pm$ & $2.9 \pm$ & $2.5 \pm$ & $2.1 \pm$ \\
& Aspergillus & 0.1 & 0.3 & 0.3 & 0.2 & 0.2 \\
2 & niger & $0.2 \pm$ & $10.4 \pm$ & $10.3 \pm$ & $1.7 \pm$ & $1.9 \pm$ \\
& Aspergillus & $8.0 \pm$ & 0.4 & 0.5 & 0.1 & 0.1 \\
3 & flavus & 0.4 & 0.3 & $1.9 \pm$ & $2.1 \pm$ & $1.7 \pm$ \\
4 & Fusarium & $4.3 \pm$ & $7.4 \pm$ & $1.7 \pm$ & 0.1 & 0.1 \\
& redolens & 0.6 & 0.2 & 0.1 & 0.2 & $1.2 \pm$ \\
5 & Penicillium & $2.6 \pm$ & $4.3 \pm$ & $2.4 \pm$ & $5.1 \pm$ & $2.0 \pm$ \\
& spinulosum & 0.2 & 0.1 & 0.3 & 0.3 & 0.3 \\
6 & Penicillium sp. & $2.7 \pm$ & $3.4 \pm$ & $5.4 \pm$ & $8.9 \pm$ & $7.1 \pm$ \\
& & 0.1 & 0.2 & 0.2 & 0.5 & 0.4 \\
\hline
\end{tabular}

Note: Mean \pm S.E. of three replicates $(n=3)$.

\subsection{Measurement of $\mathrm{pH}$ change using PDA broth}

In the present work, we have studied the $\mathrm{pH}$ variation in different time of incubation using Potato dextrose broth. In that, total five phosphate solubilizing fungal species were used for this study. Initial $\mathrm{pH}$ of all the fungal species is showed $5.6 \pm 0.2$. After incubation were showed the less $\mathrm{pH}$ level in all fungal isolates. The present study concentrates on phosphate solubilizing fungal organisms in mine soil such as Aspergillus niger, Aspergillusflavus, Fusarium redolens, Penicillium spinulosum and Penicillium sp. were isolated. These isolates were separately inoculated in to the Potato dextrose broth. The $\mathrm{pH}$ range of all the fungal species was initially at $5.6 \pm 0.2$ and finally variation of $\mathrm{pH}$ was observed. Control showed $\mathrm{pH}$ of $6.02 \pm 0.1$ at first day and finally it reaches to $5.99 \pm 0.5$ in fifth day of incubation as followed by Aspergillus niger showed $\mathrm{pH}$ of $3.88 \pm 0.2$ at first day and finally it reaches to $6.18 \pm 0.4$ in fifth day, Aspergillusflavus showed $\mathrm{pH}$ of $3.87 \pm 0.1$ at first day and finally it reaches to $\mathrm{pH} 6.0 \pm 0.3$ in fifth days, respectively (Table 6).

Table 6: Phosphate Solubilizing Ability of the Selected Five Fungal Species Studied $\mathrm{pH}$ Variation in Different Time of Incubation Using Potato Dextrose Broth

\begin{tabular}{llllllll}
\hline $\begin{array}{l}\text { SI. } \\
\text { No. }\end{array}$ & $\begin{array}{l}\text { Name of } \\
\text { the } \\
\text { organism }\end{array}$ & $\begin{array}{l}\text { Intial } \\
\mathrm{pH}\end{array}$ & $\begin{array}{l}1^{\text {st }} \\
\text { day }\end{array}$ & $\begin{array}{l}2^{\text {nd }} \\
\text { day }\end{array}$ & $\begin{array}{l}3^{\text {rd }} \\
\text { day }\end{array}$ & $\begin{array}{l}4^{\text {th }} \\
\text { day }\end{array}$ & $\begin{array}{l}5^{\text {th }} \\
\text { day }\end{array}$ \\
\hline \multirow{2}{*}{1} & Control & $5.6 \pm$ & 6.02 & 4.05 & 5.18 & 5.70 & 5.99 \\
& & 0.2 & \pm 0.1 & \pm 0.2 & \pm 0.5 & \pm 0.2 & \pm 0.5 \\
2 & Aspergillus & $5.6 \pm$ & 3.88 & 3.70 & 4.63 & 5.85 & 6.18 \\
& niger & 0.1 & \pm 0.2 & \pm 0.2 & \pm 0.3 & \pm 0.3 & \pm 0.4 \\
3 & Aspergillus & $5.6 \pm$ & 3.87 & 3.90 & 5.78 & 5.87 & $6.0 \pm$ \\
& flavus & 0.2 & \pm 0.1 & \pm 0.1 & \pm 0.2 & \pm 0.3 & 0.3 \\
4 & Fusarium & $5.6 \pm$ & 4.28 & 3.73 & 5.81 & $6.0 \pm$ & 6.29 \\
& redolens & 0.3 & \pm 0.1 & \pm 0.2 & \pm 0.2 & 0.2 & \pm 0.3 \\
5 & Penicillium & $5.6 \pm$ & 4.02 & 5.72 & 5.60 & 5.73 & 5.82 \\
& spinulosum & 0.4 & \pm 0.3 & \pm 0.3 & \pm 0.1 & \pm 0.1 & \pm 0.2 \\
6 & Penicillium & $5.6 \pm$ & 4.46 & 4.16 & 4.35 & 4.22 & 4.74 \\
& sp. & 0.2 & \pm 0.2 & \pm 0.4 & \pm 0.2 & \pm 0.3 & \pm 0.1 \\
\hline
\end{tabular}

Note: Mean \pm S.E. of three replicates $(n=3)$

\subsection{Dual culture method}

Results showed that all the five fungi tested in this study exhibited antagonistic activities against Colletotrichum sp. The pathogen of Colletotrichum sp. radial growth of the pathogen was considerably hindered by all the test antagonists under the conditions of this study. In all cases the test fungi were found to grow more or less faster than the pathogen. Aspergillus niger $(60 \pm 13 \mathrm{~mm})$ showed growth of inhibition against Colletotrichum as followed by 
Aspergillus flavus $(65 \pm 11 \mathrm{~mm})$, Fusarium redolens $(50 \pm 9.8 \mathrm{~mm})$, Penicillium spinulosum $(30 \pm 8.7 \mathrm{~mm})$ and Penicillium sp. $(10 \pm$ 2.5mm) (Table 7).

Table 7: Antagonistic Effect of Phosphate Solubilizing Fungi against Colletotrichum Sp.

\begin{tabular}{lllll}
\hline $\begin{array}{l}\text { SL. } \\
\text { No. }\end{array}$ & $\begin{array}{l}\text { Name of the } \\
\text { organism }\end{array}$ & $\begin{array}{l}\text { Growth of } \\
\text { Organisms } \\
\text { in Control } \\
\text { Plate } \\
(\mathrm{mm})\end{array}$ & $\begin{array}{l}\text { Growth of } \\
\text { Organisms in } \\
\text { Dual Plate } \\
\text { Against Colleto- } \\
\text { trichum sp. }(\mathrm{mm})\end{array}$ & $\begin{array}{l}\text { \% of } \\
\text { Growth } \\
\text { Inhibition }\end{array}$ \\
\hline 1 & $\begin{array}{l}\text { Aspergillus niger } \\
70 \pm 10\end{array}$ & $60 \pm 13$ & $14.28 \pm 1.6$ \\
2 & $\begin{array}{l}\text { Aspergillus } \\
\text { flavus }\end{array}$ & $80 \pm 12$ & $65 \pm 11$ & $21.42 \pm 1.2$ \\
3 & $\begin{array}{l}\text { Fusarium } \\
\text { redolens }\end{array}$ & $60 \pm 11$ & $50 \pm 9.8$ & $16.66 \pm 2.0$ \\
4 & $\begin{array}{l}\text { Penicillium } \\
\text { spinulosum }\end{array}$ & $50 \pm 13$ & $30 \pm 8.7$ & $40 \pm 3.0$ \\
5 & Penicillium sp. & $30 \pm 9.4$ & $10 \pm 2.5$ & $66.66 \pm 6.0$ \\
\hline
\end{tabular}

\subsection{Effect of phosphofungi on seed germination and seedling vigour}

All the fungi reduce the seed germination significantly over untreated control but decrease in seed germination dependent on concentration of pathogen. However concentration of pathogen was increased germination of seed was reduced. It can be studied by two different seed such as Groundnut, and Green gram.

\subsubsection{Green gram}

In green gram seed control, percentage of seed germination was $100 \pm 1.0$ and seedling vigour was $2010 \pm 45$. Treated with Aspergillus niger percentage of seed germination was $100 \pm 2.1$ and seedling vigour was $2670 \pm 65$ as followed by Aspergillus flavus percentage of seed germination was $100 \pm 1.8$ and seedling vigour was $2670 \pm 60$, Fusarium redolens $100 \pm 1.1$ and seedling vigour was $2450 \pm 46$, respectively (Table 8 ).

Table 8: Comparison of Shoot Length, Root Length, Percentage of Seed Germination and Seedling Vigour of Green Gram

\begin{tabular}{|c|c|c|c|c|c|}
\hline $\begin{array}{l}\text { SI. } \\
\text { NO }\end{array}$ & Treatment & $\begin{array}{l}\text { Average } \\
\text { Root } \\
\text { Length } \\
(\mathrm{cm})\end{array}$ & $\begin{array}{l}\text { Average } \\
\text { Shoot } \\
\text { Length } \\
(\mathrm{cm})\end{array}$ & $\begin{array}{l}\% \text { of Seed } \\
\text { Germination }\end{array}$ & $\begin{array}{l}\text { Seedling } \\
\text { Vigour } \\
\text { Index }\end{array}$ \\
\hline 1 & Control & $\begin{array}{l}6.0 \pm \\
0.21\end{array}$ & $\begin{array}{l}14.1 \pm \\
2.5\end{array}$ & $100 \pm 1.0$ & $\begin{array}{l}2010 \pm \\
45\end{array}$ \\
\hline 2 & $\begin{array}{l}\text { Aspergillus } \\
\text { niger }\end{array}$ & $\begin{array}{l}6.9 \pm \\
0.24\end{array}$ & $\begin{array}{l}19.8 \pm \\
2.8\end{array}$ & $100 \pm 2.1$ & $\begin{array}{l}2670 \pm \\
65\end{array}$ \\
\hline 3 & $\begin{array}{l}\text { Aspergillus } \\
\text { flavus }\end{array}$ & $\begin{array}{l}7.3 \pm \\
1.0\end{array}$ & $\begin{array}{l}16.9 \pm \\
3.1\end{array}$ & $100 \pm 1.8$ & $\begin{array}{l}2420 \pm \\
60\end{array}$ \\
\hline 4 & $\begin{array}{l}\text { Fusarium } \\
\text { redolens. }\end{array}$ & $\begin{array}{l}10.0 \pm \\
1.4\end{array}$ & $\begin{array}{l}14.5 \pm \\
1.6\end{array}$ & $100 \pm 1.1$ & $\begin{array}{l}2450 \pm \\
46\end{array}$ \\
\hline 5 & $\begin{array}{l}\text { Penicillium } \\
\text { spinulosum. }\end{array}$ & $\begin{array}{l}6.2 \pm \\
0.46\end{array}$ & $\begin{array}{l}17.6 \pm \\
1.9\end{array}$ & $100 \pm 2.0$ & $\begin{array}{l}2380 \pm \\
55\end{array}$ \\
\hline 6 & $\begin{array}{l}\text { Penicillium } \\
\text { species }\end{array}$ & $\begin{array}{l}6.6 \pm \\
0.13\end{array}$ & $\begin{array}{l}17.2 \pm \\
2.2\end{array}$ & $100 \pm 3.0$ & $\begin{array}{l}2380 \pm \\
58\end{array}$ \\
\hline
\end{tabular}

Note: Mean \pm S.E. of three replicates $(n=3)$.

\subsubsection{Ground nut seed}

In ground nut seed control, percentage of seed germination was $100 \pm 0.81$ and seedling vigour was $1020 \pm 47$. Treated with Aspergillus niger percentage of seed germination was $44 \pm 2.4$ and seedling vigour was $573 \pm 20$ as followed by Aspergillus flavus percentage of seed germination was $48 \pm 3.2$ and seedling vigour was $540 \pm 30$, Fusarium redolenspercentage of seed germination was $50 \pm 4.4$ and seedling vigour was $455 \pm 25$, respectively (Table 9).
Table 9: Comparison of Shoot Length, Root Length, Percentage of Seed Germination and Seedling Vigour of Ground Nut.

\begin{tabular}{llllll}
$\begin{array}{l}\text { S1. } \\
\text { No. }\end{array}$ & Treatment & $\begin{array}{l}\text { Average } \\
\text { Root } \\
\text { Length } \\
(\mathrm{cm})\end{array}$ & $\begin{array}{l}\text { Average } \\
\text { Shoot } \\
\text { Length } \\
(\mathrm{cm})\end{array}$ & $\begin{array}{l}\text { \% of Seed } \\
\text { Germination }\end{array}$ & $\begin{array}{l}\text { Seedling } \\
\text { Vigour } \\
\text { Index }\end{array}$ \\
\hline \multirow{2}{*}{1} & Control & $\begin{array}{l}4.2 \pm \\
1.4\end{array}$ & $\begin{array}{l}6.0 \pm \\
1.3\end{array}$ & $100 \pm 0.81$ & $\begin{array}{l}1020 \pm \\
47\end{array}$ \\
& Aspergillus & $6.0 \pm$ & $6.9 \pm$ & $44 \pm 2.4$ & $567.6 \pm$ \\
2 & niger & 1.3 & 1.4 & & 20 \\
& Aspergillus & $5.5 \pm$ & $5.7 \pm$ & $48 \pm 3.2$ & $537.6 \pm$ \\
3 & flavus & 1.0 & 1.5 & & 30 \\
4 & Fusarium & $3.5 \pm$ & $5.6 \pm$ & $50 \pm 4.4$ & $455 \pm 25$ \\
& redolens & 1.1 & 1.1 & & $122.4 \pm$ \\
5 & Penicillium & $1.6 \pm$ & $1.8 \pm$ & $36 \pm 4.5$ & 10 \\
& spinulosum. & 0.4 & 5.4 & & $190 \pm 15$ \\
6 & Penicillium & $4.3 \pm$ & $5.2 \pm$ & $20 \pm 3.2$ & \\
& sp. & 1.2 & 1.1 & &
\end{tabular}

Note: Mean \pm S.E. of three replicates $(n=3)$.

\subsection{Production of Indole 3 acetic acid using Phosphate solubilizing fungi}

In the present study, we have also studied the production of Indole-3-acetic acid by Phosphate solubilizing fungi. In that Aspergillus flavus showed the positive result (brown-red colour) and remain four fungal species are not showed the Indole-3-acetic acid (Figure 2) (Table 10).

Table 10: Production of Indole Acetic Acid Using Phosphate Solubilizing Fungi

\begin{tabular}{lll}
\hline S1.No. & Phosphate solubilizing fungi & Indole-3-Acetic Acid (IAA) \\
\hline 1 & Aspergillus flavus (AF) & $\begin{array}{l}\text { Positive } \\
\text { (Red brown colour) }\end{array}$ \\
2 & Aspergillus niger (AN) & Negative \\
3 & Fusarium redolens (FR) & Negative \\
4 & Penicillium spinulosum (PS) & Negative \\
5 & Penicillium sp. (P) & Negative \\
\hline
\end{tabular}

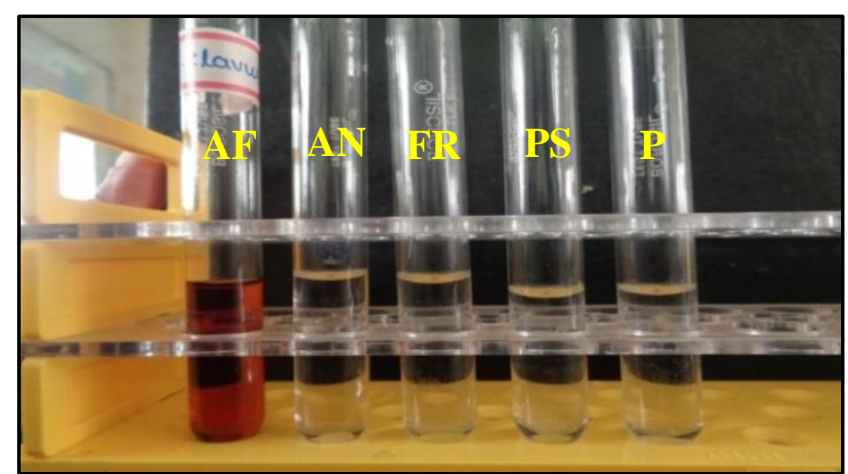

Fig. 1: Production of Indole-3 Acetic Acid (IAA) from Phosphate Solubilizing Fungi (Aspergillus Flavus Showed the Positive Result, Red Brown Colour Indicates the IAA Production).

Note: AF- Aspergillus flavus, AN- Aspergillus niger, FR- Fusarium redolens, PS- Penicillium spinulosum, P- Penicillium sp.

\section{Discussion}

Fungi have been reported to possess greater ability to solubilize rock-phosphate than bacteria (Illmer et al., 1995; Nelofer et al., 2009). The fungi, and probably all living organisms, synthesize a number of phosphatases which are necessary to scavenge phosphates $(\mathrm{Pi})$ from medium containing bound phosphorus. Both acid and alkaline phosphatases exist in soil and are distinguished on the basis of $\mathrm{pH}$ ranges at which they are active. These are secreted in response to signals of the absence of Pi (Peleg et al., 1996; Oteino et al., 2016). Seed or soil inoculation with PSMs is known to i prove solubilization of fixed soil $\mathrm{P}$ and applied phosphates, resulting in higher crop yields (El-Komy, 2005; Rudresh et al., 2005). The present study deals with isolation and characterization of phosphate solubilizing fungi using Aspergillus niger, Aspergillus 
flavus, Fusarium redolens, Penicillium spinulosum and penicillium sp. were isolated from the mine soil. Karpagam and Nagalakshmi, (2014) reported that phosphate solubilizing microbes plays an important role in plant nutrition through increase in phosphate uptake by plants and used as biofertilizers of agricultural crops. Phosphate is one of the most vital macronutrient required for the growth and development of plants. A large number of microorganisms present in the rhizosphere are known to solubilize and make available the insoluble phosphorus in the available form to the plants.

In the present study, phosphate solubilizing isolated fungi showed solubilizing activities as detected in Pikovskaya's agar medium by the appearance of halo zones around the inoculum on the medium. Isolates of organisms isolated from mine soil in this method, A. niger showed maximum level of phosphate solubilizing activity compared to other four phosphofungi. Because then medium was supplemented with tricalcium phosphate (TCP) all organisms utilizes this TCP as sole source of phosphorous and produces halo zones (Pradhan and Sukla, 2005; Malviya et al., 2011; Ruangsanka, 2014).

In this study $\mathrm{pH}$ was no affected to the medium. The Penicillium sp. showed maximum reducing $\mathrm{pH}$ in the medium compared to other organisms. It showed initial $\mathrm{pH}$ is 7 and final $\mathrm{pH}$ is 4.74 . Gupta et al., (2010) reported that Penicillium sp. showed more $\mathrm{pH}$ change. The decreasing $\mathrm{pH}$ indicates a large number of microorganisms are known to produce acidic metabolites and chelation of metal ions release fixed or insoluble phosphorous in available form.

In this study, the fungal filtrate was taken and titrates against $0.01 \mathrm{~N} \mathrm{NaOH}$ using phenolphthalein as an indicator. In first day of titration Aspergillus flavus consume more amount of $0.01 \mathrm{NNaOH}$ compare to other organisms. In fifth day of titration Penicillium sp. consumes more amount of $0.01 \mathrm{~N} \mathrm{NaOH}$.

The solubilizing activity was checked by quantitative method, the third day of incubation Aspergillus niger showed maximum solubilizing activity compared to other organisms. In $12^{\text {th }}$ day of incubation period showed more solubilizing activity by P. spinulosum and Penicillium sp. Same relevant work done by Naveenkumar et al., (2012) reported that A. niger showed maximum solubilizing activity and yield of biomass than compared to other organisms. In the present study, the Penicillium sp. showed a maximum growth of inhibition compared to other phosphofungi against Colletotrichum sp. Ajith and Lakshmidevi, (2010) reported that the antagonistic activity of Z. masonii was studied against Colletotrichum capsici, pathogen responsible for Anthracnose disease in bell pepper, by dual culture and poisoned food technique in-vitro. Seed vigour index and pot experiments were also conducted by treating Capsicum seeds with culture filtrate of $\mathrm{Z}$. masonii under greenhouse conditions. Formation of clear inhibition zone in dual culture and decrease in mycelial growth of pathogen were observed when treated with volatiles and non-volatile compounds from the antagonist. Z. masonii treated seeds showed significant increase in seed germination, shoot length, root length and dry weight of the plant. The experiments showed that $\mathrm{Z}$. masonii is a potential antagonist to control anthracnose and can be used as a biocontrol agent.

In the current study, the results showed that the inoculation of the green gram seeds with inoculation of phosphofungi, significantly increased seed germination rate and vigour index than compared to control. But in ground nut seeds, control showed more seed germination rate and vigour index than compared to treated phosphofungi. It indicates these phosphofungi are more effective for growth of green gram seeds than compared to ground nut seeds. In the present work, all phospho fungi do not produced Indole acetic acid except A. flavus. Indole acetic acid is a type of auxin that essential for plant growth and development. IAA involved in various plant physiological processes such as root initiation, cell elongation, vascular tissue differentiation and flowering Salkowski reagent used in production of IAA assay serves as an indicator of the IAA biosynthesis. This reagent reacts with Indole pyruvic acid that accumulates in the filtrate resulting in the formation of pink color (Kafrawi et al., 2014).

Potentials of phosphate solubilizing fungi could be effectively exploited in the future for the production of eco-friendly phosphate solubilizing biofertilizer for sustainable agriculture.

\section{Conclusion}

Present study had revealed that mine soil serve as good source for the growth of phosphate solubilising fungi which were grown on special pikovskaya media in the presence of tricalcium phosphate as a source of inorganic phosphorous. The phosphate solubilising fungi has been accelerating for both ecological and economic reasons. Primarily for its use as alternative to chemical phosphorous fertilizer and production beneficial phosphofungi from rhizosphere soil resources may improve the soil fertility, enhance the plant growth and reduce the risk of environmental pollution and diminish accumulation of phosphorous. Five fungal strains i.e. Aspergillus niger, Aspergillus flavus, Fusarium redolens, Penicillium spinulosum and Penicillium sp. could solubilize tricalcium phosphate. Their efficient phosphate solubilization ability, it is acceptable to propose here that these fungal strains have plant growth potentials and could be exploited as biofertilizers or bioinoculant.

\section{Acknowledgements}

Authors are gratefully acknowledged to the Department of Microbiology, Kuvempu University, Shivamogga, Karnataka, India, for providing necessary facilities and support for the completion of this work. They are indebted to their family and friends for their good wishes for the success of this work.

\section{References}

[1] Ajith PS \& Lakshmidevi N (2010) Effect of volatile and nonvolatile compounds from Trichoderma spp. against Colletotrichum capsici incitant of Anthracnose on Bell peppers. Nature and Science 8, 265-269.

[2] Aneja KR (2001) Experiments in microbiology, plant pathology and biotechnology. 4th ed. New age international publishers, New Delhi pp. 157-162.

[3] Anjalisoni, SejalRokad \& Sharma P (2013) Screening of efficient halotolerant phosphate solubilizing bacteria and their effect on seed germination under saline conditions. Journal of Scientific and Innovative Research 5, 932-937.

[4] Anjoram ST \& Mohammed M (2014) Effect of seed-borne fungi on germination and seedling vigour of watermelon (Litrulluslanatus thumb). African Journal of Plant Science 8, 232-236. http://dx.doi.org/10.5897/AJPS08.090.

[5] Ashok VG, Sabina SS \& Preeti GD (2012) Isolation and identification of phosphate solubilizing fungi from rhizosphere (soil). International Journal of science Innovation and Discoveries 2, 310-315.

[6] Baig M, Fatima S, Kadam VB \& Shaikh Y (2012) Utilization of antagonist seed borne fungi. Trends in life sciences 1, 43-46. http://dx.doi.org/10.3923/ja.2006.600.604.

[7] Doni F, Anizan CZM, CheRadziah \& Salman AH (2014) Enhancement of rice seed germination and vigour by Trichoderma sp. Research Journal of applied Science Engineering and technology 7, 4547-4552. http://dx.doi.org/10.19026/rjaset.7.832.

[8] Elias F, Woyessa D \& Muleta D (2016) Phosphate solubilization potential of rhizosphere fungi isolated from plants in Jimma Zone, Southwest Ethiopia. International Journal of Microbiology, pp. 111. http://dx.doi.org/10.1155/2016/5472601

[9] El-Komy HMA (2005) Coimmobilization of Azospirillum lipoferum and Bcillus megaterium for successful phosphorus and nitrogen nutrition of wheat plants. Food Technology and Biotechnology 43, 19-27.

[10] Funder S (1961) Practical mycology manual for identification of fungi. A.W. Broggers Boktrykkari A/S, Norway.

[11] Gupta N, Das S \& Basak UC (2010) TCP and rock phosphate solubilization by mangrove fungi grown under different $\mathrm{pH}$ and tem- 
perature in liquid culture. Journal of Agricultural technology 6, 421-428.

[12] Gupta M, Kiran S,Gulatic A, Singh B \& Tewaria R (2012) Isolation and identification of phosphate solubilizing bacteria able to enhance the growth and aloin-A biosynthesis of Aloe barbadensis miller. Microbiological Research, 167, 358-363. http://dx.doi.org/10.1016/j.micres.2012.02.004.

[13] Illmer P, Barbato A \& Schinner F (1995) Solubilization of hardlysoluble $\mathrm{AlPO}_{4}$ with $\mathrm{P}$-solubilizing microorganisms. Soil Biology and Biochemistry 27, 265-270. http://dx.doi.org/10.1016/0038-0717(94)00205-F.

[14] Kafrawi, Bahuruddin, Ennyl, Sengin \& Rosmana A (2014) Screening of free-living Indole acetic acid producing Rhizobacteria from shallot rhizospheres in the Island of Sulawesi. International Journal of Scientific and Technology Research 3, 118-121.

[15] Kannahi M \& Umaragini N (2013) Isolation characterization and antagonistic effect of phosphate solubilising microorganisms from Vignaradiata L. Rhizospheric soil. International Journal current microbiology and applied science 2, 83-88.

[16] Kapri A \& Tewari L (2010) Phosphate solubalization potential and phosphatase activity of rhizospheric Trichoderma spp. Brazilian Journal of Microbiology 41, 787-795. http://dx.doi.org/10.1590/S1517-83822010005000001.

[17] Karpagam T \& Nagalakshmi PK (2014) Isolation and characterization of phosphate solubilizing microbes from agricultural soil. International Journal of Current Microbiology and Applied Sciences 3, 601-614.

[18] Karunai S, Paul JAJ, Ravindra AD \& Vijaya V (2011) Quantitative estimation of insoluble inorganic phosphate solubilization. International Journal of Science and Nature 2, 292-295.

[19] Kulkarni GB, Sanjeevkumar, Kirankumar M, Santoshkumar \& Karegoudar TB (2011) Indole-3-Acetic acid biosynthesis in Fusarium delphinoides Strain GPK, a causal agent of Wilt in Chickpea. Applied Biochemistry and Biotechnology 169, 12921305. http://dx.doi.org/10.1007/s12010-012-0037-6.

[20] Kumar ACS, Choudhary, Paswan D, Kumar B \& Anjanaarun (2014) Sustainable way for enhancing phosphorus efficiency in agricultural soils through phosphate solubilizing microbes. An Asian Journal of Soil Science, 9, 300-310. http://dx.doi.org/10.15740/HAS/AJSS/9.2/300-310.

[21] Malviya J, Singh K \& Joshi V (2011) Effect of phosphate solubilizing fungi on growth and nutrient uptake of ground (Arachishypogaea) Plants. Advances in Bioresearch 3, 110-113.

[22] Manikandan S \& Muthuselvam K (2014) Inter strain difference of phosphor bacterial isolates of Bhendi rhizosphere for their growth promoting traits under in vitro conditions. International Journal of Current microbiology and Applied Sciences 3, 193-202.

[23] Morales A, Alvear M, Valenzuela E, Castillo CE \& Borie F (2011) Screening, evaluation and selection of phosphate-solubilising fungi as potential biofertiliser. Journal of Soil Science and Plant Nutrition11, 89-103. http://dx.doi.org/10.4067/S0718-95162011000400007.

[24] Naik SK, Maurya S, Riteshkumar JS, Das CB \& Kumar S (2013) Evaluation of rhizospheric fungi from acid soils of Jharkand on phosphate solubilization. The Bioscan 8, 875-880.

[25] Naveenkumar KJ, Thippeswamy B, Banakar SP \&Thirumalesh BV (2012) Lignolytic and phosphate solubilizing efficiency of fungal species isolated from areca nut husk waste. Journal of Research in Biology 2,143-151.

[26] Nelofer R, Syed Q, Nadeem M, Bashir F, Mazhar S \& Hassan A (2009) Solubilization of organic and inorganic phosphates by three highly efficient soil bacterial isolates. World Journal of Microbiology and Biotechnology 25, 1471-1477. http://dx.doi.org/10.1007/s11274-009-0037-z

[27] Nisha K, Devi SNP, Vasandha S \& Sunithakumari K (2014) Role of phosphate solubilizing microorganism to eradicate $\mathrm{p}$ - deficiency in plant. International Journal of Scientific and Research Publications 4, 2250-3153.

[28] Oteino N, Lally RD, Kiwanuka S, Lloyd A, Ryan D, Germaine KJ \& Dowling DN (2016) Plant growth promotion induced by phosphate solubilising endophytic Pseudomonas isolates. Frontiers in Microbiology 6, 1-9. http://dx.doi.org/10.3389/fmicb.2015.00745.

[29] Paul JJA \& Daniel T (2007) Lignolytic and phosphate solubilizing efficiency of fungal species isolated from Municipal solid waste. Asian Journal of Microbiology, Biotechnology and Environmental Sciences 9, 837-840.

[30] Peleg Y, Addison R, Aramaya R \& Metzenberg RL (1996) Translocation of Neurospora crassa transcription factor NUC-1 into the nucleus is induced by phosphate limitation. Fungal Genetics and Biology 20, 185-191. http://dx.doi.org/10.1006/fgbi.1996.0034.

[31] Ponmurugan P \& Gopi C (2006) Distribution Pattern and Screening of Phosphate solubilizing bacteria isolated from different food and forage crops. Journal of Agronomy 5, 600-604. http://dx.doi.org/10.3923/ja.2006.600.604.

[32] Pooja KS, Naveen Kumar KJ \& Lokesh ST (2015) Isolation and screening of efficient phosphate solubilizing fungi from forest soil. Inventi Rapid: Agro Tech 4, 1-7.

[33] Pradhan N \& Sukla LB (2005) Solubilization of inorganic phosphates by fungi isolated from agriculture soil. African Journal of Biotechnology 5, 850-854.

[34] Priya S, Panneerselvam T \& Sivakumar T (2013) Evaluation of indole-3-acetic acid in phosphate solubilizing microbes isolated from rhizosphere soil. International Journal of Current Microbiology and Applied Science 2, 29-36.

[35] Ruangsanka S (2014) Identification of phosphate-solubilizing fungi from the asparagus rhizosphere as antagonists of the root and crown rot pathogen Fusarium oxysporum. Science Asia, 40, 16-20. http://dx.doi.org/10.2306/scienceasia1513-1874.2014.40.016.

[36] Rudresh DL, Shivaprakash MK \& Prasad RD (2005) Tricalcium phosphate solubilizing abilities of Trichoderma spp. In relation to $\mathrm{P}$ up take and growth yield parameters of chickpea (Cicerarietinum L.). Canadian Journal of Microbiology 51, 217-226. http://dx.doi.org/10.1139/w04-127.

[37] Sadasivam M \& Manickam A (1996) Biochemical methods. 2nd ed. New age international Limited Publishers, New Delhi pp.1-235.

[38] Sembiring M, Elfiati D, Sutarta ES \& Sabrina T (2015) Effect of Talaromyces pinophilus and SP36 on phosphate available and Potato (Solanum tuberosum L) production on andisol impacted by Mount Sinabung Eruption, North Sumatera, Indonesia. International Journal of Sciences: Basic and Applied Research 24, 382-388.

[39] Srilatha P \& Venkateshwarlu K (2009) Potential of Rhizosphere microorganisms in phosphate solubilization. Ecology, Environment and Conservation 15,115-119.

[40] Subramanian CV (1993) Hyphomycetes taxonomy and biology. 1st ed. Academic Press, London pp. 1-930. 